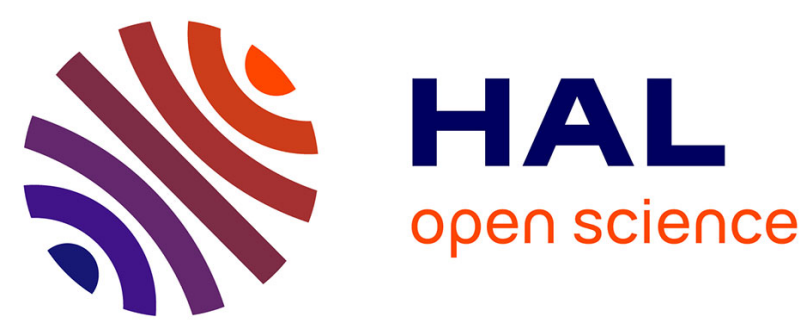

\title{
Investigation of the Optical and Excitonic Properties of the Visible Light-Driven Photocatalytic BiVO4 Material
}

Tilak Das, Xavier Rocquefelte, Robert Laskowski, Luc Lajaunie, Stéphane Jobic, Peter Blaha, Karlheinz Schwarz

\section{- To cite this version:}

Tilak Das, Xavier Rocquefelte, Robert Laskowski, Luc Lajaunie, Stéphane Jobic, et al.. Investigation of the Optical and Excitonic Properties of the Visible Light-Driven Photocatalytic BiVO4 Material. Chemistry of Materials, 2017, 29 (8), pp.3380-3386. 10.1021/acs.chemmater.6b02261 . hal-01532179

HAL Id: hal-01532179

https://hal-univ-rennes1.archives-ouvertes.fr/hal-01532179

Submitted on 23 Jun 2017

HAL is a multi-disciplinary open access archive for the deposit and dissemination of scientific research documents, whether they are published or not. The documents may come from teaching and research institutions in France or abroad, or from public or private research centers.
L'archive ouverte pluridisciplinaire HAL, est destinée au dépôt et à la diffusion de documents scientifiques de niveau recherche, publiés ou non, émanant des établissements d'enseignement et de recherche français ou étrangers, des laboratoires publics ou privés. 


\section{INTRODUCTION}

In 1972, Honda and Fujishima first discovered that it was possible to promote the water splitting reaction, i.e. the water decomposition into oxygen and hydrogen, by using an electrochemical device based on a $\mathrm{TiO}_{2}$ anode and a platinum counter electrode exposed to UV-light. ${ }^{1}$ Over the past four decades, an important number of investigations has been devoted to such photoelectrochemical (PEC) reactions. Namely, $\mathrm{TiO}_{2}$ is well-suited for photocatalytic applications due to its low cost, its high stability in aqueous solutions and the right positioning of its valence band maximum (VBM) and conduction band minimum (CBM). Unfortunately, due to its too large band gap $(>3 \mathrm{eV})$, it operates only in the UV range and thus considerably reduces the efficiency of the solar to hydrogen conversion. To have efficiencies above $15 \%$ a band gap below $2.2 \mathrm{eV}$ is required ${ }^{2}$. One strategy to reach this band gap requirement is to consider multiternary oxides combining $\mathrm{nd}^{\mathrm{o}}$ (e.g. $\mathrm{Ti}^{4^{+}}, \mathrm{V}^{5+}, \mathrm{Nb}^{5+}$ ) and $\mathrm{ns}^{2}$ cations (e.g. $\mathrm{Bi}^{3+}$, $\mathrm{Sn}^{2+}$ ). Then, energy levels can be interspaced between the former valence and conduction bands and thus favor the absorption in the visible range. In that context, bismuth vanadate $\left(\mathrm{BiVO}_{4}\right)$, initially known for its ferroelastic and ionic conductivity properties ${ }^{3,4,5}$, is now considered as one of the most promising candidate for solar-to-hydrogen conversion processes operating in the visible light range ${ }^{6,7,8}$. However, its properties strongly depend on the allotropic form of $\mathrm{BiVO}_{4}$. Three main polymorphs are known, namely the zircon structure with tetragonal symmetry $\left(Z_{T}-\mathrm{BiVO}_{4}\right)$ and two (almost identical) scheelite structures with monoclinic $\left(S_{M^{-}}-\mathrm{BiVO}_{4}\right)$ and tetragonal $\left(S_{T^{-}}\right.$ $\mathrm{BiVO}_{4}$ ) symmetries, the former being ferroelastic, the latter paraelastic ${ }^{9} . S_{M^{-}}$and $S_{T}-\mathrm{BiVO}_{4}$ were selectively prepared by many groups showing that $S_{M}-B_{i V O}$ exhibits high photoelectrochemical (PEC) properties, whereas the photoactivity of $Z_{T}-\mathrm{BiVO}_{4}$ is negligible ${ }^{6,7,9}$. It has also been demonstrated that both $\mathrm{S}_{\mathrm{M}^{-}}$and $\mathrm{S}_{\mathrm{T}^{-}}-\mathrm{BiVO}_{4}$ structures are photocatalytic active for the degradation of rhodamine $\mathrm{B}$ in solution under visible light, but not $\mathrm{Z}_{\mathrm{T}}-\mathrm{BiVO}_{4}{ }^{10}{ }^{10}$

Recently, H. Fan et al. ${ }^{11}$ shed light on the higher PEC activity of $S_{M^{-}-B i V O_{4}}$ compared to $Z_{T}-B_{i V O}$. Based on surface photovoltage and transient photovoltage measurements under visible light irradiation, the authors clearly evidenced that photo-induced electrons (holes) flow from the surface (bulk) to the bulk (surface) for $\mathrm{Z}_{\mathrm{T}}-\mathrm{BiVO}_{4}$ while the opposite is observed for $\mathrm{S}_{\mathrm{M}}-\mathrm{BiVO}_{4}$. This explains why $\mathrm{S}_{\mathrm{M}}-\mathrm{BiVO}_{4}$ is more active than $\mathrm{Z}_{\mathrm{T}}-\mathrm{BiVO}_{4}$ for oxidation reactions. In addition, R. Li et al. ${ }^{12}$ have evidenced that, under photo-irradiation, efficient charge separation is achieved in $\mathrm{S}_{\mathrm{M}}-\mathrm{BiVO}_{4}$, leading to a reduction reaction with photogenerated electrons and oxidation reaction with photo- 
generated holes on the $\{010\}$ and $\{110\}$ crystal facets, respectively.

In order to address the differences between $S_{M^{-}}, S_{T^{-}}$and $Z_{T}-\mathrm{BiVO}_{4}$ the optical properties have been investigated. Up to date, only the optical band gaps of $S_{M^{-}}, S_{T^{-}}$and $Z_{T^{-}}$ $\mathrm{BiVO}_{4}$ have been estimated based on diffuse reflectance spectra on powder samples, and no experimental investigation on their frequency dependent dielectric function (DF) has been reported so far. From the theoretical side, a significant number of studies has been carried out using density functional theory (DFT) with the generalized gradient approximation (GGA) and hybrid functionals. However, contradictory results have been published. For instance, the band gap nature of $S_{M^{-}}-\mathrm{BiVO}_{4}$ was reported both as direct ${ }^{2,13}$ and indirect ${ }^{14,15}$. Moreover, two different groups have reported simulations of the frequency dependent dielectric function ${ }^{14,15}$, suggesting that the optical anisotropy is strong in $\mathrm{S}_{\mathrm{M}}-\mathrm{BiVO}_{4}$, and negligible in $\mathrm{Z}_{\mathrm{T}^{-}}$ $\mathrm{BiVO}_{4}{ }^{14}$.

Clearly, all these previous studies of the optical properties of $\mathrm{BiVO}_{4}$ suffer from being purely experimental or theoretical. An investigation of the optical and excitonic properties of $\mathrm{BiVO}_{4}$ combining experiments and theory in order to raise our understanding of the photocatalytic activity of this material is still missing. This is, indeed, the main purpose of the present work, which reports, for the very first time, on the local-field, optical anisotropy and excitonic effects in $S_{M^{-}}$and $Z_{T}-\mathrm{BiVO}_{4}$, based on electron energy loss spectroscopy (EELS) measurements, groundstate DFT calculations including crystal local-field effects $^{16}$ (LFE) and many-body corrections using the Bethe Salpeter equation $^{17}$ (BSE). An excellent agreement is obtained between EELS experiments and DFT calculations, which fully validates such $a b$ initio calculations as a starting point for more sophisticated calculations devoted to the excitonic properties. In particular, solving the BetheSalpeter equations (BSE) allows studying the spatial distribution of the photo-induced electron-hole pairs, i.e. the excitons, in $\mathrm{S}_{\mathrm{M}^{-}}$and $\mathrm{Z}_{\mathrm{T}}-\mathrm{BiVO}_{4}$ phases, and evidences the impact of the long-range crystalline structure on it. From the present work, a unified picture of the optical and excitonic properties of $\mathrm{S}_{\mathrm{M}^{-}}$and $\mathrm{Z}_{\mathrm{T}^{-}} \mathrm{BiVO}_{4}$ is proposed which emphasizes the link between the nature of band gap, the photocatalytic activity, and the excitonic properties. Finally, this result is supported by a comparison of two materials, namely $\mathrm{TiO}_{2}$ anatase and rutile, which are well known for their differences in the photocatalytic property, being important and negligible, respectively.

\section{RESULTS AND DISCUSSION}

Structurally speaking, $\mathrm{S}_{\mathrm{M}^{-}}$and $\mathrm{S}_{\mathrm{T}^{-}} \mathrm{BiVO}_{4}$ are very simi$\operatorname{lar}^{18}$, except a small distortion, which reduces the symmetry from tetragonal (space group $\mathrm{I}_{4} / \mathrm{a}$ for $\mathrm{S}_{\mathrm{T}}$, with $\mathrm{a}=\mathrm{b}$ $=5.147$ and $\mathrm{c}=11.7216 \AA$ ) to monoclinic (space group $\mathrm{I} 2 / \mathrm{b}$ for $S_{M}$, with $a=5.1935, b=5.0898$ and $\left.c=11.6972 \AA\right)$. In contrast, the zircon phase ${ }^{19}\left(\mathrm{Z}_{\mathrm{T}}-\mathrm{BiVO}_{4}\right.$ - space group $\mathrm{I}_{4}$ /amd, with $\mathrm{a}=\mathrm{b}=7.303$ and $\mathrm{c}=6.584 \AA$ ) is significantly different from the two scheelite phases, leading to a large volume expansion to $87.8 \AA^{3} /$ f.u. compared to the $\mathrm{S}_{\mathrm{M}^{-}}$and
$\mathrm{S}_{\mathrm{T}^{-}}$phases, with a volume of 77.6 and $77.3 \AA^{3} /$ f.u., respectively. Using the following setting, i.e. $\mathrm{I}_{4} / \mathrm{amd}, \mathrm{I}_{4} / \mathrm{a}$ and I2/b space groups, for $\mathrm{Z}_{\mathrm{T}^{-}}, \mathrm{S}_{\mathrm{T}^{-}}$and $\mathrm{S}_{\mathrm{M}^{-}} \mathrm{BiVO}_{4}$, respectively, it is possible to compare these three allotropes, with a common definition of the c-axis and the (a,b)-plane. Such a setting will be used all along the manuscript, allowing to discuss optical anisotropy and excitonic spatial distribution using a common setting for the crystallographic directions for the $\mathrm{BiVO}_{4}$ phases. Both scheelite $\left(\mathrm{S}_{\mathrm{T}}\right.$ and $\left.\mathrm{S}_{\mathrm{M}}\right)$ and zircon $\left(\mathrm{Z}_{\mathrm{T}}\right)$ structures consist of isolated $\left[\mathrm{VO}_{4}\right]^{3^{-}}$ tetrahedra connected by $\left[\mathrm{BiO}_{8}\right]^{5-}$ antiprisms but differ in the way these polyhedra are connected to each other. The structural phase transition between zircon and scheelite has been evidenced to be based on a first-order reconstruction involving a "bond-switching" mechanism. ${ }^{20}$ Figures $1 \mathrm{a}$ and $\mathrm{rb}$ show the consequences of such a structural filiation between the $\mathrm{S}_{\mathrm{M}^{-}}$(or $\mathrm{S}_{\mathrm{T}^{-}}$) and $\mathrm{Z}_{\mathrm{T}^{-}} \mathrm{BiVO}_{4}$ phases. Starting from the $S_{M}$ phase, three changes are observed to reach the $Z_{T}$ phase: (1) a rotation of the $\left[\mathrm{VO}_{4}\right]^{3^{-}}$tetrahedral units; (2) an expansion along the aand b-axes of the monoclinic cell; (3) a strong compression along the c-axis. The main impact of these structural modifications concerns the interconnection between the $\left[\mathrm{VO}_{4}\right]^{3^{-}}$and $\left[\mathrm{BiO}_{8}\right]^{{ }^{-}}$units as shown in Figures ic and 1d. The $\mathrm{Bi}-\mathrm{V}$ bond lengths and $\mathrm{Bi}-\mathrm{O}-\mathrm{V}$ bond angles of $\mathrm{S}_{\mathrm{M}^{-}}$and $\mathrm{Z}_{\mathrm{T}}-\mathrm{BiVO}_{4}$ are summarized in Table $\mathrm{S}_{1}$ and will be discussed in more details below, in the section devoted to the optical anisotropy.

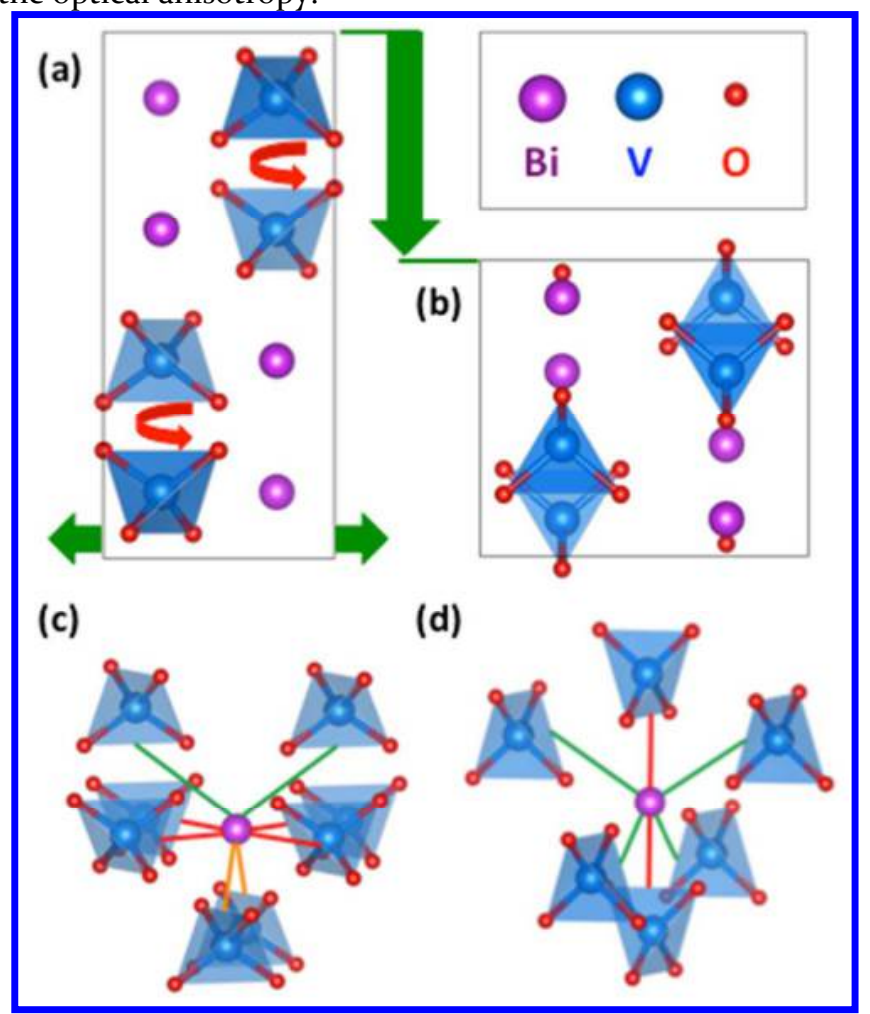

Figure 1. Schematic representation of the atomic structures of $\mathrm{S}_{\mathrm{M}^{-}}-\mathrm{BiVO}_{4}$ (a) and $\mathrm{Z}_{\mathrm{T}}-\mathrm{BiVO}_{4}$ (b). The structure filiation from $\mathrm{S}_{\mathrm{M}^{-}}$and $\mathrm{Z}_{\mathrm{T}^{-}} \mathrm{BiVO}_{4}$ is highlighted with the green arrows (cell expansion along $\mathrm{a}$ and $\mathrm{b}$, cell contraction along $\mathrm{c}$ ) and the red arrows (rotation of $\mathrm{VO}_{4}^{3-}$ tetrahedra). The resulting local environments of bismuth atoms are given for $\mathrm{S}_{\mathrm{M}}-\mathrm{BiVO}_{4}$ (c) 
and $\mathrm{Z}_{\mathrm{T}}-\mathrm{BiVO}_{4}(\mathrm{~d})$. A color code for the $\mathrm{Bi}-\mathrm{V}$ bonds is used: shortest in red, intermediate in orange, largest in green (see Table $S_{1}$ for the related values).

Before discussing our results on the optical anisotropy of these compounds, the properties of their excitons and their potential impact on the related photocatalytic properties, we first validated our theoretical approach. To do so, we have compared the calculated energy lossfunctions with the measured low-loss EELS spectra obtained from $\mathrm{S}_{\mathrm{M}}$ and $\mathrm{Z}_{\mathrm{T}}-\mathrm{BiVO}_{4}$ samples. The Density Functional Theory (DFT) calculations have been carried out by using two different codes: the Vienna Ab initio Simulation Package (VASP) ${ }^{21}$ for the energy-loss functions including LFE and the WIEN2k program package ${ }^{22}$ as starting point for BSE evaluations of the excitonic properties. Most DFT calculations were performed with the Perdew, Burke, and Ernzerhof parameterization of the GGA (PBE-GGA) of the exchange-correlation potential. ${ }^{23}$ BSE calculations were performed using a similar strategy than the one we used for $\mathrm{CuAlO}_{2}$ compound. ${ }^{24}$

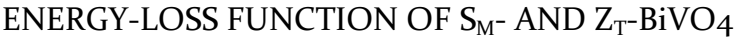

Electron energy loss spectroscopy (EELS) measurements are directly related to the energy-loss function (ELF) defined by $\operatorname{Im}[-1 / \varepsilon(v)]=\varepsilon_{2}(v) /\left(\varepsilon_{1}(v)^{2}+\varepsilon_{2}(v)^{2}\right)$, where $\varepsilon_{1}(v)$ and $\varepsilon_{2}(v)$ are the real and imaginary parts of the dielectric function, where $v$ is the frequency ${ }^{25}$. Properly combined with first-principles calculations, it is an unrivaled tool to probe the frequency dependent dielectric function over a high-energy range, ${ }^{26}$. The optical diffusion and absorption processes are directly related to $\varepsilon_{1}(v)$ and $\varepsilon_{2}(v)$, respectively. Figure 2 shows the energy loss function, $\operatorname{Im}(-1 / \varepsilon)$, of $S_{M}$ and $\mathrm{Z}_{\mathrm{T}}-\mathrm{BiVO}_{4}$. The experimental measurements have been carried out with a low collection angle, allowing a direct comparison between the recorded and calculated loss-functions at zero momentum transfer $(q \rightarrow 0)$. An excellent agreement is obtained between the present DFT calculations and orientation-dependent EELS measurements, as soon as the local-field effects (LFE) are taken into account in our simulations (more details on LFE are provided in the supplementary materials). In particular, the energy position and respective intensity each spectral feature is well-reproduced. Such an agreement between PBE-GGA calculations and EELS measurements is not expected at a first sight. Indeed, we expect an underestimation of the band gap value using PBE-GGA and thus a shift of all the simulated peaks with respect to the experimental spectrum. However, as previously reported, ${ }^{26}$ PBE-GGA simulations give correct peak positions for the high-energy part of the spectrum $(\mathrm{E}>5 \mathrm{eV})$, while the band gap underestimation has a significant impact on the low-energy part of the spectrum $(\mathrm{E}<5 \mathrm{eV})$. We are in a similar situation except that no significant spectral feature is detectable at low-energy, explaining such an overall agreement. In addition, it should be noticed that PBEGGA underestimates the band gap of $\mathrm{BiVO}_{4}$ compounds by only $0.4 \mathrm{eV}$ (see below).

We should mention that the loss-functions are nearly the same along the three crystallographic directions for
$\mathrm{S}_{\mathrm{M}}-\mathrm{BiVO}_{4}$, in contrast to $\mathrm{Z}_{\mathrm{T}}-\mathrm{BiVO}_{4}$. This observation is important and allows us to consider an average lossfunction for $\mathrm{S}_{\mathrm{M}}-\mathrm{BiVO}_{4}$, which makes the results independent of the orientation chosen during the experiments. In contrast, the comparison for $\mathrm{Z}_{\mathrm{T}}-\mathrm{BiVO}_{4}$ requires a careful definition of the orientation axis (the origin of the different features is explained in Fig. S2 in the Supplementary Materials). It also should be noted that the tail of the zero-loss peak hinders the proper extraction of information below $\sim 5 \mathrm{eV}$ (the energy resolution of the microscope is around $0.8 \mathrm{eV}$, see Supplementary Materials). As a consequence band-gap offset and excitons cannot be probed with this experimental set-up.

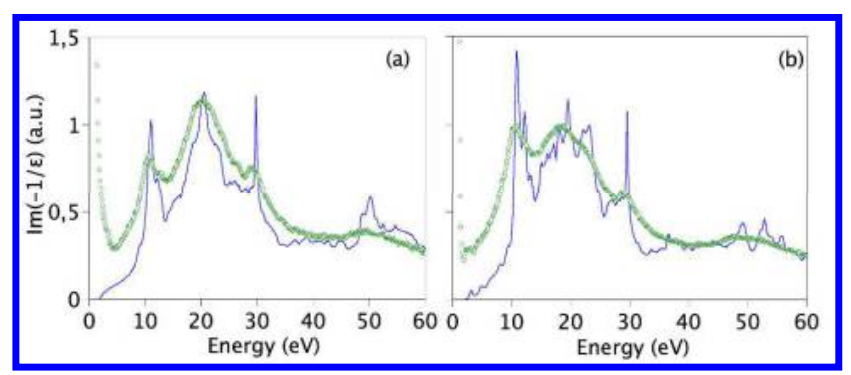

Figure 2. Energy-loss $(\operatorname{Im}(-1 / \varepsilon))$ functions of $\mathrm{S}_{\mathrm{M}}-\mathrm{BiVO}_{4}$ (a) and $\mathrm{Z}_{\mathrm{T}}-\mathrm{BiVO}_{4}$ (b). The experimental VEELS spectra (green open circles) of $\mathrm{S}_{\mathrm{M}^{-}}$and $\mathrm{Z}_{\mathrm{T}}-\mathrm{BiVO}_{4}$ have been recorded along the $\left[\begin{array}{lll}9 & 11 & 4\end{array}\right]$ and $\left[\begin{array}{lll}1 & 1 & 0\end{array}\right]$ zone axes, respectively. The calculated energy-loss and dielectric functions include local-field effects. For comparison, the calculated quantities correspond to an average of the polarizations along $\mathrm{x}, \mathrm{y}$ and $\mathrm{z}$ for $\mathrm{S}_{\mathrm{M}^{-}}$ $\mathrm{BiVO}_{4}$ and a polarization along $\mathrm{z}$ for $\mathrm{Z}_{\mathrm{T}}-\mathrm{BiVO}_{4}$.

\section{OPTICAL ANISOTROPY IN $\mathrm{BiVO}_{4} \mathrm{AND}_{\mathrm{TiO}}$}

Using a standard DFT functional appears adequate for the description of the high-energy range of the dielectric function (from 5 to $60 \mathrm{eV}$ ). However, since band gaps are strongly underestimated in conventional DFT, a more advanced method is needed for an accurate description of the low-energy range (from o to $5 \mathrm{eV}$ for instance). Consequently, we have used the Tran-Blaha modified BeckeJohnson (TB-mBJ) potential, ${ }^{27}$ which is known for its ability to describe band gaps with an accuracy comparable to sophisticated GW calculations. In our DFT calculations we have also taken into account relativistic effects on the valence electrons by including the spin-orbit (SO) coupling in our DFT calculations, to properly describe the Bi$\mathrm{p}^{1 / 2}$ and Bi- $\mathrm{p}^{3 / 2}$ states, which are involved in the bands close to the Fermi level. Indeed, a band gap reduction of about $0.1 \mathrm{eV}$ is observed when the SO coupling is switched on, for both $\mathrm{S}_{\mathrm{M}^{-}}$and $\mathrm{Z}_{\mathrm{T}^{-}}-\mathrm{BiVO}_{4}$.

Since in optical excitations the excited electron may interact with the hole it left behind, we had to go beyond standard DFT. By solving the 2-particle Bethe Salpeter Equation (BSE) one can properly describe the interaction between the electron and the hole of a photo-generated pair, i.e. the bound exciton state. Here, the starting point of the BSE calculations was not based on GW eigenvalues but on our GGA results plus a band gap correction, which was defined in such a way to reproduce the TB-mBJ and 
GW band gaps of $\mathrm{BiVO}_{4}$ and $\mathrm{TiO}_{2}$, respectively. Such an approximation of the BSE spectra leads to similar results for $\mathrm{TiO}_{2}$ than the ones starting from $\mathrm{GW}$ eigenvalues ${ }^{31}$.

From our DFT calculations (including SO coupling) we obtain an indirect and direct fundamental band gap for $\mathrm{S}_{\mathrm{M}^{-}}$and $\mathrm{Z}_{\mathrm{T}}-\mathrm{BiVO}_{4}$, which changes from 2.06 and $2.47 \mathrm{eV}$ to 2.46 and $2.84 \mathrm{eV}$ for the two phases respectively, when the TB-potential $\mathrm{mBJ}$ is taken into account. This leads to a band gap correction of about $0.4 \mathrm{eV}$ for the $\mathrm{BiVO}_{4}$ compounds.
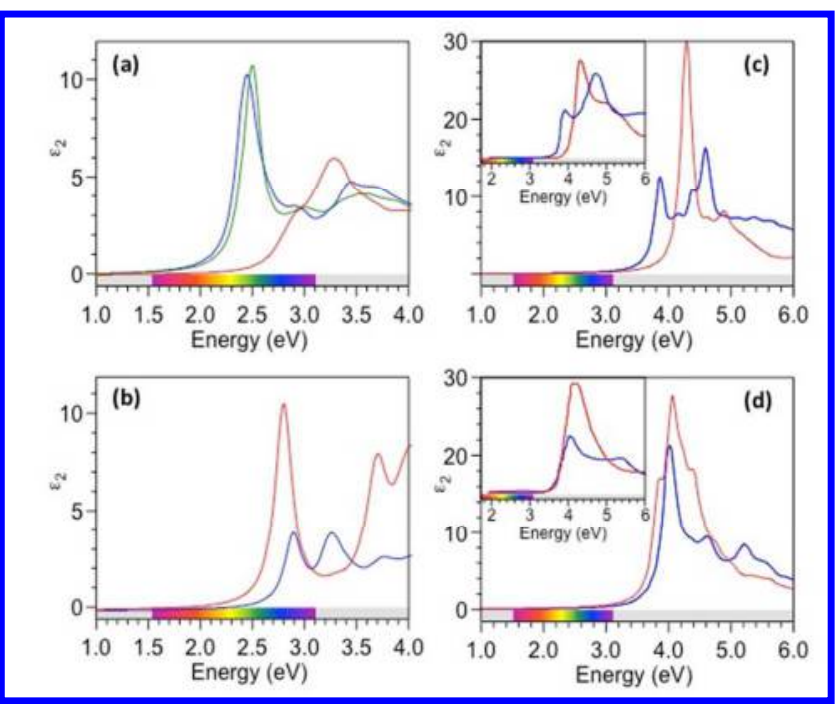

Figure 3. Imaginary part of the dielectric function for $\mathrm{S}_{\mathrm{M}}-\mathrm{BiVO}_{4}(\mathrm{a}), \mathrm{Z}_{\mathrm{T}}-\mathrm{BiVO}_{4}(\mathrm{~b})$, ana- $\mathrm{TiO}_{2}$ (c) and rut-TiO $(\mathrm{d})$ calculated via Bethe-Salpeter equation (BSE). The BSE calculated spectra have been carefully converged for the energy range shown here (i.e. up to 4 and $6 \mathrm{eV}$ for $\mathrm{BiVO}_{4}$ and $\mathrm{TiO}_{2}$, respectively). The $\mathrm{x}, \mathrm{y}$ and $\mathrm{z}$ polarizations are represented with blue, green and red solid lines. Insets: Experimental spectra for anatase- $\mathrm{TiO}_{2}$ and rutile- $\mathrm{TiO}_{2}$.

It should be noticed that the indirect nature of the band gap of $\mathrm{S}_{\mathrm{M}}-\mathrm{BiVO}_{4}$ has been recently experimentally confirmed, ${ }^{28}$ with an energy difference between the indirect $(2.56 \mathrm{eV})$ and direct $(2.68 \mathrm{eV})$ band gaps of $0.12 \mathrm{eV}$, which agrees quite well with our prediction of $0.12 \mathrm{eV} \mathrm{(2.47}$ and 2.59 eV, respectively for indirect and direct band gaps for $\mathrm{S}_{\mathrm{M}}-\mathrm{BiVO}_{4}$ ). In contrast, our calculations show a difference of $0.02 \mathrm{eV}$ between the indirect $(2.85 \mathrm{eV})$ and the direct $(2.87 \mathrm{eV})$ band gaps for $\mathrm{Z}_{\mathrm{T}}-\mathrm{BiVO}_{4}$, which is not significant.

In Figs. $3 a$ and $3 b$, the $\varepsilon_{2}$ function (in the low-energy range), deduced from BSE calculations, is plotted for $\mathrm{S}_{\mathrm{M}^{-}}$ and $\mathrm{Z}_{\mathrm{T}}-\mathrm{BiVO}_{4}$, respectively. For comparison we also show (in Figs. $3 c$ and 3 d) the $\varepsilon_{2}$ functions (based on BSE calculations) for anatase- and rutile- $\mathrm{TiO}_{2}$, respectively. $\mathrm{TiO}_{2}$ is the reference material in terms of photocatalytic performances in the UV-range, while of the two phases of $\mathrm{BiVO}_{4}$ one is a very promising candidate for photocatalysis in the visible range. In both cases, one phase (i.e. $\mathrm{S}_{\mathrm{M}}-\mathrm{BiVO}_{4}$ and anatase- $\mathrm{TiO}_{2}$ ) is known to have better photocatalytic properties than the other. Our idea is to compare these four systems and extract the main parameters that control why only two of them are efficient in the photocatalytic activity, focusing on the optical absorption and exciton formation.

The in-plane (along $\mathrm{x}$ - and/or $\mathrm{y}$-axis) and out-of-plane (along z-axis) contributions to the complex DF have been analyzed for the four afore mentioned materials. The inplane and out-of- plane notation refers to the 4 -fold axis in $\mathrm{Z}_{\mathrm{T}}-\mathrm{BiVO}_{4}$, anatase and rutile, and the 2-fold axis in $\mathrm{S}_{\mathrm{M}^{-}}$ $\mathrm{BiVO}_{4}$. Before discussing our predictions for $\mathrm{BiVO}_{4}$, let us consider the two $\mathrm{TiO}_{2}$ phases, for which experimental data are available (Figs. $3 \mathrm{C}$ and $3 \mathrm{~d}$ ). The insets of figures $3 \mathrm{C}$ and $3 \mathrm{~d}$ provide the experimental $\varepsilon_{2}$ curves of the anatase and rutile $\mathrm{TiO}_{2}$ phases obtained at room temperature ${ }^{29}$. Very good agreement is obtained with the present BSE calculations. In particular, as previously demonstrated ${ }^{30,31}$, such BSE calculations are able to reproduce the anisotropic behavior of the optical properties of anatase and rutile $\mathrm{TiO}_{2}$. The first optical excitations are from a polarization perpendicular vs. parallel to the c-axis for anatase and rutile, respectively. In other words, anatase- and rutile- $\mathrm{TiO}_{2}$ differ in their nature of the photo-excitations, where the former has two important polarizations ( $\mathrm{x}$ and y) but the latter only one $(z)$, respectively.

The optical anisotropy for $\mathrm{S}_{\mathrm{M}}-\mathrm{BiVO}_{4}$ (Fig. 3a) is very strong in the low-energy range, in contrast to our previous observation for its related loss-function in the highenergy range. The in-plane contribution leads to a band gap of about $2.4 \mathrm{eV}$, instead of $2.9 \mathrm{eV}$ for the out-of-plane contribution (see Table S1). Similarly, a strong anisotropy is found for $\mathrm{Z}_{\mathrm{T}}-\mathrm{BiVO}_{4}$ (Fig. $3 \mathrm{~b}$ ). However, the smallest optical gap is then observed for the out-of-plane contribution $(2.8 \mathrm{eV})$, while the in-plane optical gap is about 2.9 $\mathrm{eV}$. In addition, the in-plane (out-of-plane) and out-ofplane (in-plane) excitations are quite intense (low) in $\mathrm{S}_{\mathrm{M}^{-}}$ and $\mathrm{Z}_{\mathrm{T}}-\mathrm{BiVO}_{4}$. Such a difference between $\mathrm{S}_{\mathrm{M}^{-}}$and $\mathrm{Z}_{\mathrm{T}^{-}}$ $\mathrm{BiVO}_{4}$ is a direct consequence of the structural difference between these two phases, influencing the $\mathrm{V}-\mathrm{O}$ and $\mathrm{Bi}-\mathrm{O}$ interactions, which can easily be captured by the $\mathrm{Bi}-\mathrm{O}-\mathrm{V}$ bond angles and $\mathrm{Bi}-\mathrm{V}$ distances (Table $\mathrm{S} 1$ ). Indeed, the reduction of the band gap from a pure binary oxide to the ternary compounds $\mathrm{BiVO}_{4}$ is related to the presence of additional $\mathrm{Bi}-\mathrm{O}$ and $\mathrm{Bi}-\mathrm{V}$ interactions, which contribute to the anisotropy but in a different manner in the unit cell of $\mathrm{S}_{\mathrm{M}}$ and $\mathrm{Z}_{\mathrm{T}}$. To be more specific, a bond angle of $90^{\circ}$ and short $\mathrm{Bi}-\mathrm{V}$ distances are preferred to have an efficient $5 \mathrm{p}(\mathrm{Bi})-2 \mathrm{p}(\mathrm{O})-3 \mathrm{~d}(\mathrm{~V})$ interaction. These two criteria are satisfied only for the $(\mathrm{x}, \mathrm{y})$-plane in the $\mathrm{S}_{\mathrm{M}}$ phase and along the $\mathrm{z}$-axis, in the $\mathrm{Z}_{\mathrm{T}}$ phase. In summary, the optical anisotropy in $\mathrm{S}_{\mathrm{M}^{-}}$and $\mathrm{Z}_{\mathrm{T}}-\mathrm{BiVO}_{4}$ is very different at low energy, leading to an intense optical excitation in the visible range for a polarization perpendicular and parallel to the c-direction for $\mathrm{S}_{\mathrm{M}^{-}}$and $\mathrm{Z}_{\mathrm{T}}-\mathrm{BiVO}_{4}$, respectively. In other words, $\mathrm{S}_{\mathrm{M}}-\mathrm{BiVO}_{4}$ and $\mathrm{Z}_{\mathrm{T}}-\mathrm{BiVO}_{4}$ differ in their photoexcitations, which use two polarizations ( $x$ and $y$ ) in the former but one $(\mathrm{z})$ in the later compound, respectively.

We cannot conclude at this stage that such a correlation between the anisotropy of the dielectric function and the photocatalytic performances do exist, but it is interesting to note that, in both $\mathrm{TiO}_{2}$ and $\mathrm{BiVO}_{4}$, the better photocatalytic phase is the one, which shows an in-plane polar- 
ization. Additional aspects must be considered to explain the nearly zero photocatalytic activity of $\mathrm{Z}_{\mathrm{T}}-\mathrm{BiVO}_{4}$ under visible light excitation, compared to $\mathrm{S}_{\mathrm{M}^{-}}-\mathrm{BiVO}_{4}$. This difference could stem from the significantly larger band gap in the $\mathrm{Z}_{\mathrm{T}}$-phase, which in our $\mathrm{TB}-\mathrm{mBJ}$ calculations is about $2.9 \mathrm{eV}$ and thus on the very edge of energies reachable by visible light.

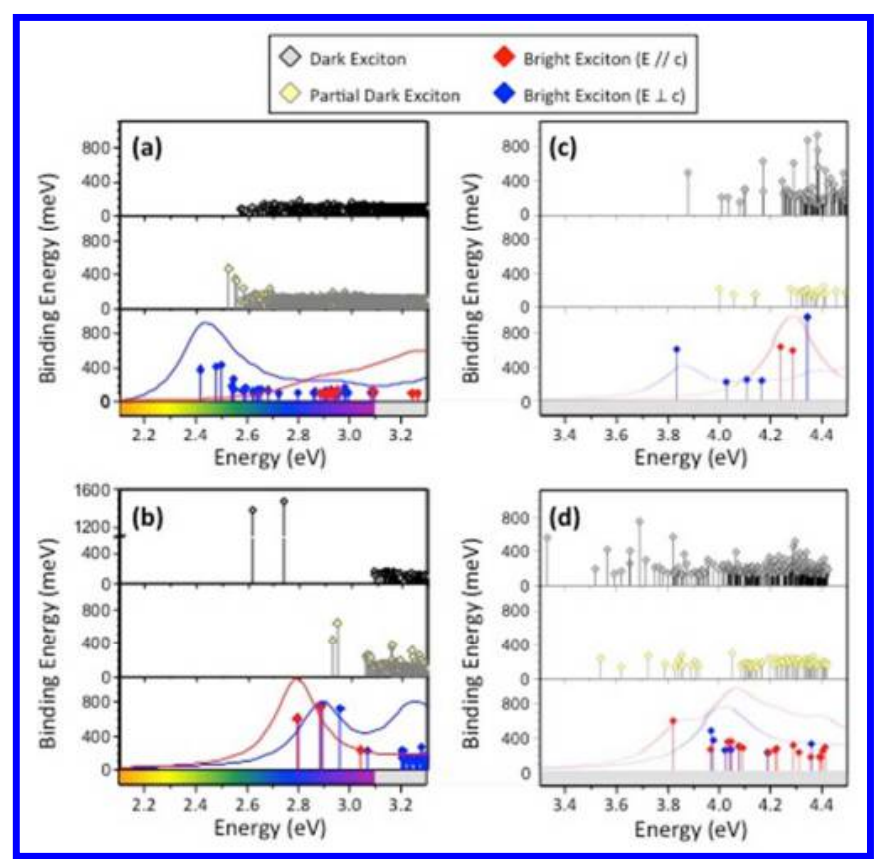

Figure 4. The first excitons calculated via the BetheSalpeter equation (BSE) are shown in three dark, partially dark or bright (i.e. oscillator strength is zero, small and large) for $\mathrm{S}_{\mathrm{M}}-\mathrm{BiVO}_{4}(\mathrm{a}), \mathrm{Z}_{\mathrm{T}}-\mathrm{BiVO}_{4}$ (b), ana-TiO (c) and rut- $\mathrm{TiO}_{2}(\mathrm{~d})$. For each exciton the binding energy is given. For the bright excitons the imaginary part of the dielectric functions is given.

\section{EXCITONIC EFFECTS IN $\mathrm{BiVO}_{4}$ AND TiO2}

An exciton is by definition a correlated electron-hole, bound by a Coulombic interaction. Figure 4 shows the first thousand excitons of the four systems and their distribution in energy. Their binding energies $\left(E_{b}\right)$ characterize their stability. A common feature is observed in the two photocatalytic active compounds, anatase- $\mathrm{TiO}_{2}$ and $\mathrm{S}_{\mathrm{M}}-\mathrm{BiVO}_{4}$. In both of these phases the first exciton is bright and polarized perpendicularly to the c-axis. This is in contrast to the inactive compounds rutile- $\mathrm{TiO}_{2}$ and $\mathrm{Z}_{\mathrm{T}^{-}}$ $\mathrm{BiVO}_{4}$, for which the first exciton is dark. In addition, the first bright exciton for rutile- $\mathrm{TiO}_{2}$ is the $24^{\text {th }}$ at $3.82 \mathrm{eV}$, with a polarization parallel to the c-axis and a strong binding energy of $605 \mathrm{meV}$.

From the present BSE calculations, we can estimate the hole-electron weighted mean distance, hereafter labeled $d_{h-e}$, which combined with $E_{b}$, the binding energy of the exciton, will be a measure for the probability of a holeelectron recombination, i.e. the exciton life-time. The quantity $\mathrm{d}_{\mathrm{h}-\mathrm{e}}$ has been estimated using the expression:

$$
\left\langle d_{h-e}\right\rangle=\frac{\sum_{h=1}^{N} \sum_{c=0}^{M} \sum_{e=1}^{N}\left(d_{h-e}^{c} . P_{h-e}^{c}\right)}{\sum_{h=1}^{N} \sum_{c=0}^{M} \sum_{e=1}^{N} P_{h-e}^{c}}
$$

where $P_{h-e}^{c}$ the probability of an hole-electron pair characterized by the distance $d_{h-e}^{c}$ is given by:

$$
P_{h-e}^{c}=\int_{r_{h}} d^{3} r_{h} \int_{r_{e}} d^{3} r_{e} \mathrm{~W}\left(r_{h}, r_{e}\right)
$$

$\mathrm{W}\left(\mathrm{r}_{\mathrm{h}}, \mathrm{r}_{\mathrm{e}}\right)$ the conditional probability of having electron at $\mathrm{r}_{\mathrm{e}}$ and hole at $\mathrm{r}_{\mathrm{h}}$ by:

$$
\mathrm{W}\left(r_{e}, r_{h}\right)=\phi\left(r_{e}, r_{h}\right) \phi^{*}\left(r_{e}, r_{h}\right)
$$

$\phi\left(r_{e}, r_{h}\right)$ and the BSE excitonic envelop function.

The three indices ( $h, c$ and e) refer as to the position of the electron (e) and the hole (h) over the $\mathrm{N}$ atomic sites in the cell defined by the index $\mathrm{c}$, where $\mathrm{c}$ ranges between o (origin cell) to M. M is taken such that the maximum he distance is about $30 \AA$ A. The calculated probabilities for larger distances are negligible. In the present calculations the hole is systematically in the cell at the origin $(c=0)$ and the bound electron in the cell c. A similar approach was used by S. Sharifzadeh et al. ${ }^{32}$ to estimate the average h-e distance in organic solids.

(a)
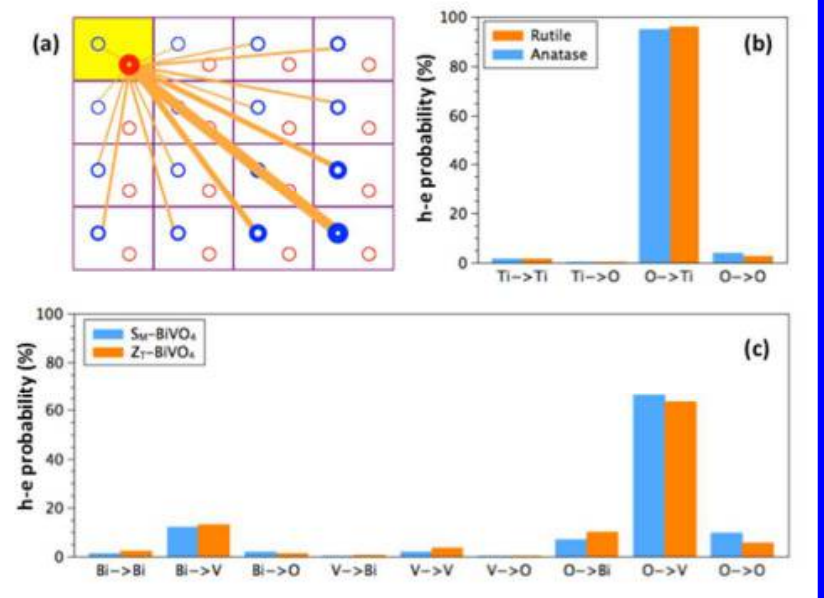

Figure 5. A schematic representation of $<\mathrm{d}_{\mathrm{h}-\mathrm{e}}>$ for a unit cell containing only two atoms (oxygen in red, metal in blue) (a), h-e probability distribution for rutile- and anatase- $\mathrm{TiO}_{2}$ (b) and $\mathrm{S}_{\mathrm{M}^{-}}$and $\mathrm{Z}_{\mathrm{T}^{-}}-\mathrm{BiVO}_{4}$ (c). The photo-hole is inside the cell at the origin (defined by 000) and the photo-electron is distributed over all cells with a given h-e probability (proportional to the line width).

Figure 5a shows a schematic representation of the way $\left\langle\mathrm{d}_{\mathrm{h}-\mathrm{e}}\right\rangle$ is estimated. For simplicity, the unit cell contains only two atoms. The cell at the origin is highlighted in yellow; the orange lines represent the h-e contacts. Here the hole is defined on the oxygen site inside the cell at the origin and the promoted electron on a metal site. The h-e probabilities are proportional to the width of the lines, i.e. the thicker the lines, the larger the probabilities to create an exciton between the two connected sites. By averaging all these weighted-distances, we can thus define $\left\langle\mathrm{d}_{\mathrm{h}-\mathrm{e}}>\right.$ based on the previous formula.

Figures $5 \mathrm{~b}$ and $5 \mathrm{c}$ show the distribution of the e-h probabilities for the first bright excitons of rutile- $\mathrm{TiO}_{2}$, anatase- $\mathrm{TiO}_{2}, \mathrm{~S}_{\mathrm{M}}-\mathrm{BiVO}_{4}$ and $\mathrm{Z}_{\mathrm{T}}-\mathrm{BiVO}_{4}$. It clearly shows that, in both rutile- and anatase- $\mathrm{TiO}_{2}$, about $95 \%$ of the excitons occur between oxygen and titanium atoms (the se- 
cond largest $\mathrm{P}_{\mathrm{e}-\mathrm{h}}$ is very small and corresponds to transitions between oxygen atoms due to hybridization effects). In $\mathrm{BiVO}_{4}$, the main contribution to the exciton spectra originates from optical transitions between oxygen and vanadium $(\mathrm{O} \rightarrow \mathrm{V}$, i.e. hole on $\mathrm{O}$ and electron on $\mathrm{V})(66$ and $63 \%$ of the transitions for $\mathrm{S}_{\mathrm{M}}-\mathrm{BiVO}_{4}$ and $\mathrm{Z}_{\mathrm{T}}-\mathrm{BiVO}_{4}$, respectively), much more than the bismuth to vanadium transitions (with 12 and $13 \%$ relative weight for $\mathrm{S}_{\mathrm{M}}-\mathrm{BiVO}_{4}$ and $\mathrm{Z}_{\mathrm{T}}-\mathrm{BiVO}_{4}$, respectively). Finally, contributions $\mathrm{O} \rightarrow \mathrm{Bi}$ and $\mathrm{O} \rightarrow \mathrm{O}$ are also observed but can be neglected. Moreover, while in $\mathrm{TiO}_{2}$ the photo-hole was located nearly exclusively on oxygen, in $\mathrm{BiVO}_{4}$ it is mainly positioned on oxygen but partly also on bismuth (more than $10 \%$ ). Similarly, in $\mathrm{TiO}_{2}$ the photo-electron is nearly exclusively located on the metal center (titanium). In contrast, in the vanadate ternary compounds it is distributed on vanadium (a little less than $80 \%$ with two transitions $\mathrm{O} \rightarrow \mathrm{V}$ and $\mathrm{Bi} \rightarrow \mathrm{V}$ ), bismuth (due to $\mathrm{O} \rightarrow$ Bi transition, with 7 and $10 \%$, in $\mathrm{S}_{\mathrm{M}}-\mathrm{BiVO}_{4}$ and $\mathrm{Z}_{\mathrm{T}}-\mathrm{BiVO}_{4}$, respectively), and oxygen (due to $\mathrm{O} \rightarrow \mathrm{O}$ transition, with 10 and $6 \%$, in $\mathrm{S}_{\mathrm{M}}-\mathrm{BiVO}_{4}$ and $\mathrm{Z}_{\mathrm{T}}-\mathrm{BiVO}_{4}$, respectively). Such an excitonic distribution is the direct consequence of the orbital overlap in these compounds as illustrated in Figure $\mathrm{S}_{3}$ for $\mathrm{Z}_{\mathrm{T}}-\mathrm{BiVO}_{4}$.
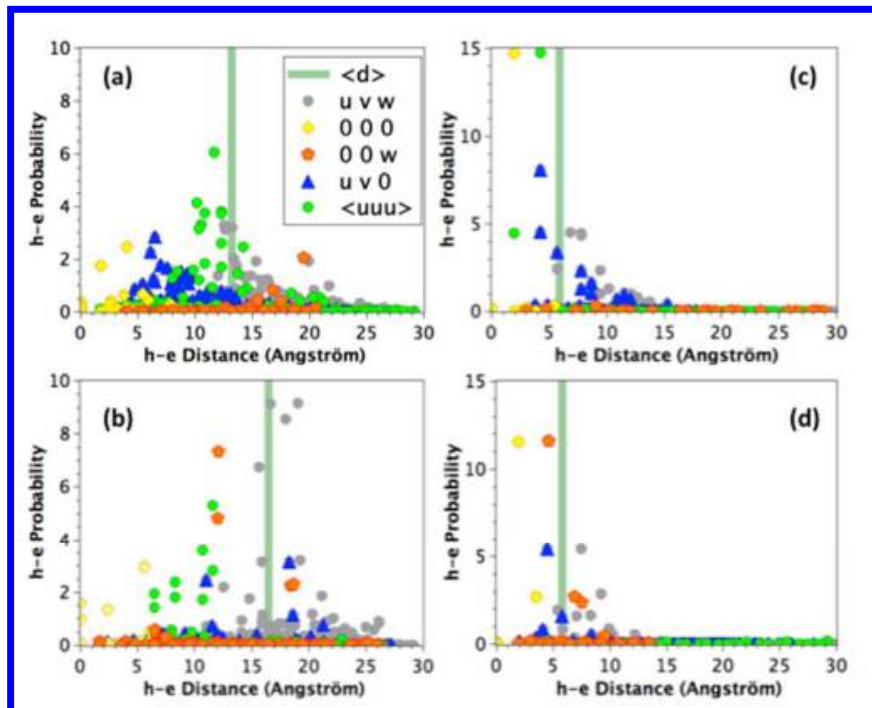

Figure 6 . The h-e distance distribution of the first bright excitons for $\mathrm{S}_{\mathrm{M}}-\mathrm{BiVO}_{4}(\mathrm{a}), \mathrm{Z}_{\mathrm{T}}-\mathrm{BiVO}_{4}(\mathrm{~b})$, ana-TiO ${ }_{2}$ (c) and rut- $\mathrm{TiO}_{2}(\mathrm{~d})$. It corresponds to excitons \#1 (at $2.41 \mathrm{eV} / \mathrm{E}_{\mathrm{b}}$ $=384 \mathrm{meV}), \#_{3}\left(\right.$ at $\left.2.79 \mathrm{eV} / \mathrm{E}_{\mathrm{b}}=610 \mathrm{meV}\right), \#_{1}($ at $3.84 \mathrm{eV} /$ $\mathrm{E}_{\mathrm{b}}=608 \mathrm{meV}$ ) and \#24 (at $3.82 \mathrm{eV} / \mathrm{E}_{\mathrm{b}}=605 \mathrm{meV}$ ), respectively for $\mathrm{S}_{\mathrm{M}}-\mathrm{BiVO}_{4}, \mathrm{Z}_{\mathrm{T}}-\mathrm{BiVO}_{4}$, anatase-TiO${ }_{2}$ and rutile- $\mathrm{TiO}_{2}$. For each compound the green bar highlights the mean h-e distance.

The optimal situation to avoid the electron-hole recombination is to have a small $E_{b}$ and a large $d_{h-e}$. Based on the examination of average $d_{h-e}$ and $E_{b}$ values, we plot in Figure 6 the probability that an electronic transition occurs vs. its associated $\mathrm{d}_{\mathrm{h}-\mathrm{e}}$ distance. Only the first bright excitons of the four compounds are taken into account. Among all the photo-excitations (represented with grey circles), some specific ones are highlighted. The ones corresponding to excitons localized in the unit cell are represented with yellow squares. The ones corresponding to an exciton that propagates in [?]0],[00?] and <uuu> directions, are depicted by blue triangles, orange pentagons and green circles, respectively. Finally a green bar highlights the previously calculated average $d_{h-e}$ distance in each material for the given exciton. Note the similarities between $\mathrm{S}_{\mathrm{M}^{-}}$and $\mathrm{Z}_{\mathrm{T}}-\mathrm{BiVO}_{4}$, both in terms of $\mathrm{d}_{\mathrm{h}-\mathrm{e}}$ distance and the shape of the h-e distribution. In $\mathrm{S}_{\mathrm{M}}-\mathrm{BiVO}_{4}$, $\mathrm{Z}_{\mathrm{T}}-\mathrm{BiVO}_{4}$ and anatase $\mathrm{TiO}_{2}$, the excitons which propagate along <uuu> directions have high probabilities. In contrast the highest probability in rutile $\mathrm{TiO}_{2}$ is found for excitons propagating along the $[00 \mathrm{w}]$ direction. Figure 7 gives a very simple interpretation by showing the projection of the four structures along specific directions. Indeed, it appears that the exciton will propagate mainly in the direction of the highest packing of $\mathrm{Bi}$ in both $\mathrm{BiVO}_{4}$ phases, which is along the <uuu> directions, and along the direction where the $\mathrm{TiO}_{6}$ octahedrons are sharing their edges, which is along <uuu> and [00? directions for anatase and rutile- $\mathrm{TiO}_{2}$, respectively.

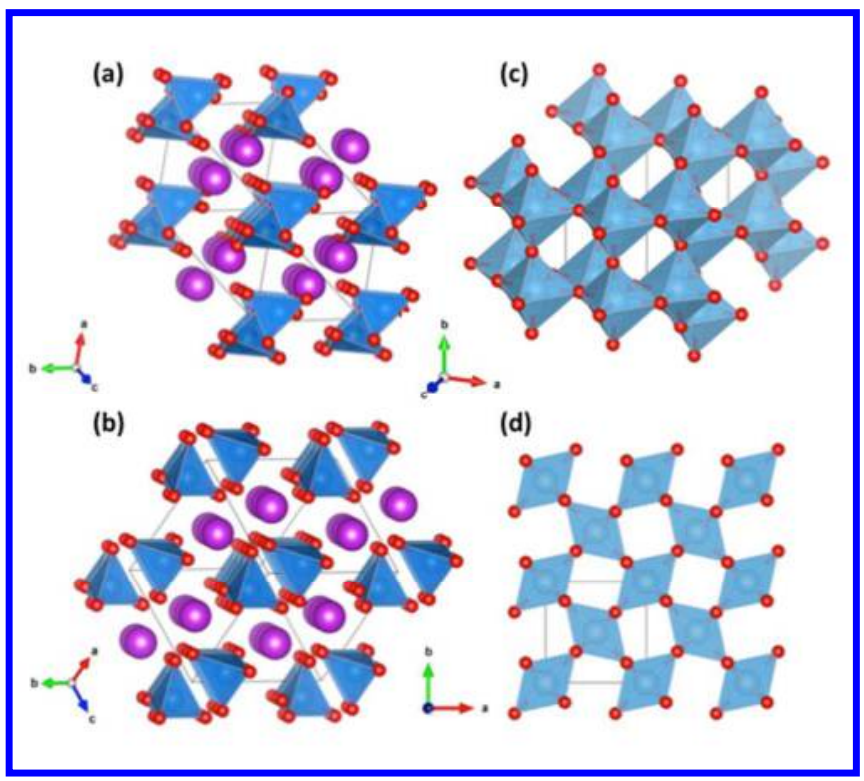

Figure 7. Projection of the atomic structure along the highest e-h probability direction for $\mathrm{S}_{\mathrm{M}^{-}}-\mathrm{BiVO}_{4}(\mathrm{a}), \mathrm{Z}_{\mathrm{T}^{-}}$ $\mathrm{BiVO}_{4}(\mathrm{~b})$, ana-TiO $(\mathrm{c})$ and rut $-\mathrm{TiO}_{2}$ (d). It corresponds to the <111> direction for (a), (b) and (c) and the [oo1] direction for (d).

Another way to analyze the present BSE data is to consider the Supplementary Tables $\mathrm{S}_{1-4}$, which gives the excitation energy, the oscillator strength and the binding energy of the principal bright excitons in the four systems. For instance, considering the first fifty excitons, 13 are bright in $\mathrm{S}_{\mathrm{M}^{-}}-\mathrm{BiVO}_{4}$, but only 5 in $\mathrm{Z}_{\mathrm{T}}-\mathrm{BiVO}_{4}$. Similarly, among the first fifties excitons, 10 are bright in anatase$\mathrm{TiO}_{2}$, while only 1 in rutile $\mathrm{TiO}_{2}$. In summary, under visible light excitation, more electron-hole pairs are formed in $\mathrm{S}_{\mathrm{M}^{-}}$than in $\mathrm{Z}_{\mathrm{T}}-\mathrm{BiVO}_{4}$, and these excitons have the propensity to recombine more frequently in $\mathrm{Z}_{\mathrm{T}^{-}}$than in $\mathrm{S}_{\mathrm{M}^{-}}$ $\mathrm{BiVO}_{4}$. A similar analysis could be done for anatase- and rutile- $\mathrm{TiO}_{2}$.

\section{CONCLUSION}


In conclusion, the present investigation evidences many common features between the reference UV-light photocatalytic compound anatase- $\mathrm{TiO}_{2}$ and the promising candidate for visible-light photocatalytic compound $\mathrm{S}_{\mathrm{M}^{-}}$ $\mathrm{BiVO}_{4}$. Indeed, both exhibit an indirect band gap, their first exciton is bright and shows a polarization perpendicular to the c-axis. The fact that the band gap of the two photocatalytic active materials is indirect is essential in the sense that it prevents a direct recombination of the hole-electron pair, leading to longer lifetime of photoexcited electrons and holes than direct band gap rutile- $\mathrm{TiO}_{2}$ and $\mathrm{Z}_{\mathrm{T}}-\mathrm{BiVO}_{4}$. In addition, the first excitons of these two last compounds are dark and show a polarization parallel to the c-axis. These inter-related properties appear to be essential and should be considered with care in the quest of an optimal photocatalytic material.

\section{AUTHOR INFORMATION}

\section{Corresponding Author}

TOC GRAPHIC

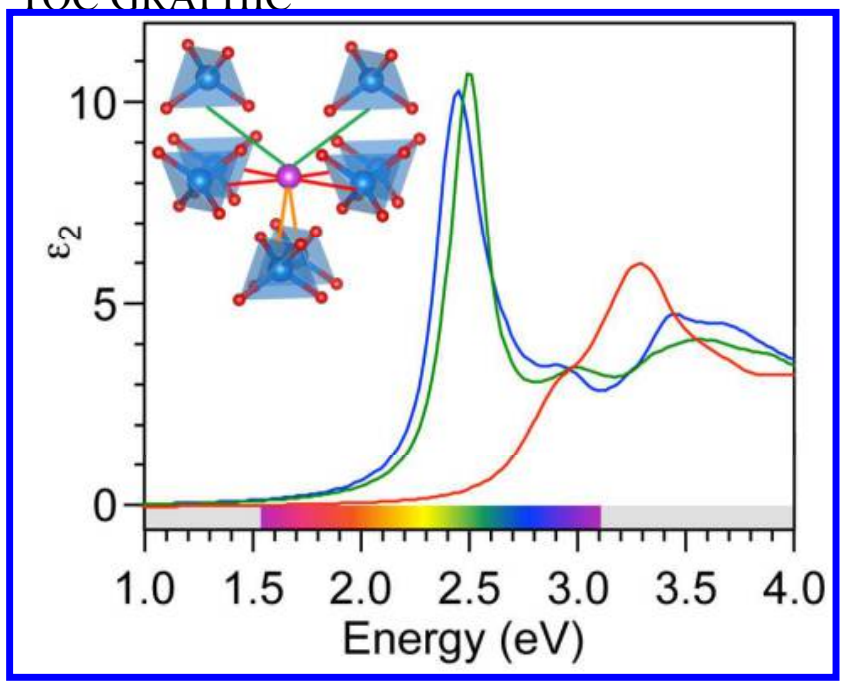

*Address correspondence to xavier.rocquefelte@univrennesı.fr

\section{Author Contributions}

XR and SJ initiated the project, LL performed the EELS measurements and their analyses, TD, PB and XR performed the DFT simulations, RL performed the BSE calculations. All authors analyzed the data and wrote the paper and contributed to corrections and modifications.

\section{ACKNOWLEDGMENT}

X.R. thanks T. Saison for the synthesis of $\mathrm{S}_{\mathrm{M}^{-}} \mathrm{BiVO}_{4}$ and $\mathrm{Z}_{\mathrm{T}^{-}}$ $\mathrm{BiVO}_{4}$ and the CCIPL (Nantes) computing centre for computational facilities. P.B. was supported by the Austrian Science Foundation FWF (SFB F41 'Vicom'). LL acknowledges the support received from the European Union Seventh Framework Program under Grant Agreement 312483 ESTEEM2 (Integrated Infrastructure Initiative - I3) and from the European Union H2020 program under the grant 696656 Graphene Flagship

1. Fujishima, A.; Honda, K. Electrochemical Photolysis of Water at a Semiconductor Electrode Nature 1972, $238,37-38$.

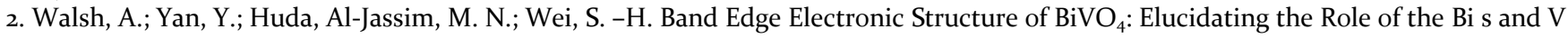
d Orbitals Chem. Mater. 20o9, 21, 547-551.

3. Yeom, T. H.; Choh, S. H.; Song, K. J.; Jang, M. S. The domain structure of ferroelastic $\mathrm{BiVO}_{4}$ studied by magnetic resonances J. Phys.: Condens. Matter. 1994, 6, 383-392.

4. Yeom, T. H. The domain structure of ferroelastic $\mathrm{BiVO}_{4}$ crystal studied by Gd ${ }^{3+}$ EPR Solid State Communications 2oo3, 125, 547-550. 5. Manolikas, C.; Amelinckx, S. Ferroelastic domains in $\mathrm{BiVO}_{4}$ Phys. Stat. Sol. (a) 2oo6, 6o, 167-170.

6. Kudo, A.; Omori, K.; Kato, H. A Novel Aqueous Process for Preparation of Crystal Form-Controlled and Highly Crystalline BiVO 4 Powder from layered Vanadates at Room Tempetarure and Its Photocatalytic and Photophysical Properties J. Am. Chem. Soc. 1999, 121, $11459-11467$.

7. Zhang, X.; Ai, Z.; Jia, F.; Zhang, L.; Fan, X.; Zou, Z. Selective synthesis and visible light photocatalytic activity of $\mathrm{BiVO}_{4}$ with different crystalline phases Mater Chem. Phys. 20o7, 103, 162-167.

8. Li, C.; Pang, G.; Sun, S.; Feng, S. Phase transition of BiVO4 nanoparticles in molten salt and the enhancement of visible-light photocatalytic activity J. Nanopart. Res. 2010, 12, 3069-3075.

9. Tokunaga, S.; Kato, H.; Kudo, A. Selective Preparation of Monoclinic and Tetragonal $\mathrm{BiVO}_{4}$ with Scheelite Structure and Their Photocatalytic Properties Chem. Mater. 20o1, 13, 4624-4628. 
10. Saison, T.; Chemin, N.; Chanéac, C.; Durupthy, O.; Mariey, L.; Maugé, F.; Brezova, V.; Jolivet, J.P. New Insights Into BiVO ${ }_{4}$ Properties as Visible Light Photocatalyst J. Phys. Chem. C 2015, 119, 12967.

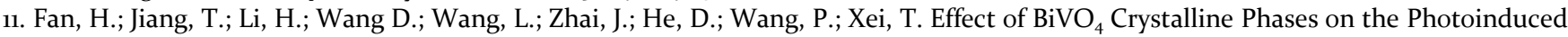
Carriers Behavior and Photocatalytic Activity J. Phys. Chem. C 2012, 116, 2425-2430.

12. Li, R.; Zhang, F.; Wang, D.; Yang, J.; Li, M.; Zhu, J.; Zhou, X.; Han, H.; Li, C. Spatial separation of photogenerated electrons and holes among $\{010\}$ and $\{110\}$ crystal facets of $\mathrm{BiVO}_{4}$ Nat. Commun. 2013, 4, 1432.

13. Kweon, K. E.; Hwang, G. S. Hybrid density functional study of the structural, bonding, and electronic properties of bismuth vanadate Phys. Rev. B 2012, 86, 165209.

14. Zhao, Z.; Li, Z.; Zou, Z. Electronic structure and optical properties of monoclinic clinobisvanite $\mathrm{BiVO}_{4}$ Phys. Chem. Chem. Phys. 2o11, 13, $4746-4753$.

15. Wadnerkar, N.; English, N.J. Density theory functional investigations of bismuth vanadate: Effect of hybrid functionals Comput. Mater. Sci. 2013, 74, 33-39.

16. Aryasetiawan, F.; Gunnarsson, O.; Knupfer, M.; Fink, J. Local-field effects in NiO and Ni Phys. Rev. B 1994, 50, 7311.

17. Sham, L. J.; Rice, T. M. Many-Particle Derivation of the Effective-Mass Equation for the Wannier Exciton Phys. Rev. 1966, 144, 708714 .

18. Sleight, A. W.; Chen, H. -Y.; Ferretti, A.; Cox, D. E. Crystal growth and structure of BiVO4 Materials Research Bulletin 1979, 14, 15711581.

19. Dreyer, G.; Tillmanns, E. Dreyerite: ein naturliches, tetragonales wismutvanadat von Hirschhorn/Pfalz, NeuesJahrbuch fur Mineralogie, Monatshefte 1981, 1981, 151-154.

20. Smirnov, M. B.; Mirgorodsky, Kazimirov, V. Yu.; Guinebretière, R. Bond-switching mechanism for the zircon-scheelite phase transition Phys. Rev. B 2015, 78, 094109.

21. Kresse, G.; Furthmuller, J. Efficient iterative schemes for ab-initio total-energy calculations using a plane-wave basis set Phys. Rev. $B$ 1996, 54, 11169 .

22. Blaha, P.; Schwarz, K.; Madsen, G. K. H.; Kvasnicka, D.; Luitz, J. WIEN2k, An Augmented Plane Wave Plus Local Orbitals Program for Calculating Crystal Properties (Vienna University of Technology, Austria, 2001).

23. Perdew, J. P.; Burke, K.; Ernzerhof, M. Generalized Gradient Approximation Made Simple Phys. Rev. Lett. 1996, 77, 3865-3868.

24. Laskowski, R.; Christensen, N. E.; Blaha, P.; Palanivel, B. Strong excitoniceffects in $\mathrm{CuAlO}_{2}$ delafossite transparent conductive oxides Phys. Rev. B 2009, 79, 165209.

25. Ritchie, R. H. Plasma Losses by Fast Electrons in Thin Films Phys. Rev. 1957, 106, 874.

26. Lajaunie, L.; Boucher, F.; Dessapt, R.; Moreau, P. Strong anisotropic influence of local-field effects on the dielectric response $\alpha-\mathrm{MoO}_{3}$ Phys. Rev. B 2013, 88, 115141.

27. Tran, F.; Blaha, P. Accurate Band Gaps of Semiconductors and Insulators with a Semilocal Exchange-Correlation Potential Phys. Rev. Lett. 2009, 102, 226401.

${ }^{28}$ Cooper, J.K.; Gul, S.; Toma, F.M.; Chen, L.; Liu, Y.S.; Guo, J.; Ager, J.W.; Yano, J.; Sharp, I.D. Indirect Bandgap and Optical Properties of Monoclinic Bismuth Vanadate J. Phys. Chem. C 2015, 119, 2969.

29. Cardona, M.; Harbeke, G. Optical Properties and Band Structure of Wurtzite-Type Crystals and Rutile Phys. Rev. 1965, 137, A1467. 30. Kang, W.; Hybertsen, M. S. Quasiparticle and optical properties of rutile and anatase $\mathrm{TiO}_{2}$ Phys. Rev. B 2010, 82, 085203 .

31. Chiodo, L.; Garcia-Lastra, J. M.; Lacomino, A.; Ossicini, S.; Zhao, J.; Petek, H.; Rubio, A. Self-energy and excitonic effects in the electronic and optical properties of $\mathrm{TiO}_{2}$ crystalline phases. Phys. Rev. B 2010, 82, 045207.

32 Sharifzadeh, S.; Darancet, P.; Kronik, L.; Neaton, J.B. Low-Energy Charge-Transfer Excitons in Organic Solids from First-Principles: The Case of Pentacene J. Phys. Chem. Lett. 2013, 4, 2197-2201. 